University of Massachusetts Amherst

ScholarWorks@UMass Amherst

Chemistry Department Faculty Publication Series

Chemistry

2013

\title{
Trace determination of total mercury in rice by conventional inductively coupled plasma mass spectrometry
}

\author{
Lindsay R. Drennan-Harris \\ University of Massachusetts Amherst \\ Sirinapa Wongwilawan \\ Naresuan University \\ Julian Tyson \\ University of Massachusetts Amherst
}

Follow this and additional works at: https://scholarworks.umass.edu/chem_faculty_pubs

Part of the Chemistry Commons

\section{Recommended Citation}

Drennan-Harris, Lindsay R.; Wongwilawan, Sirinapa; and Tyson, Julian, "Trace determination of total mercury in rice by conventional inductively coupled plasma mass spectrometry" (2013). Journal of Analytical Atomic Spectrometry. 1353.

Retrieved from https://scholarworks.umass.edu/chem_faculty_pubs/1353 


\title{
Trace determination of total mercury in rice by conventional inductively coupled plasma mass spectrometry
}

\author{
Lindsay R. Drennan-Harris, ${ }^{a}$ Sirinapa Wongwilawan ${ }^{\mathrm{b}}$ and Julian F. Tyson*a \\ Mercury is a potent neurotoxin with which food and beverages may be contaminated from a number of \\ sources, both natural and anthropogenic. The determination of mercury at concentrations close to \\ instrumental detection limits suffers from problems related to memory effects and loss, both during \\ sample preparation and within sample introduction systems. L-cysteine (1\%) was added to rice samples, \\ standards, and rinse solutions in order to keep the mercury in solution and decrease the memory effect. \\ Gold $\left(1 \mu \mathrm{g} \mathrm{L}^{-1}\right)$ was added online as an internal standard to improve accuracy and precision, while \\ further decreasing the memory effect. A comparison of methods involving microwave digestion or acid \\ extraction showed that both were capable of detecting single-digit $\mu \mathrm{g} \mathrm{kg}^{-1}$ concentrations of mercury \\ in rice. The microwave digestion ICP-MS procedure was further validated by a comparison of results \\ with those obtained with a solid-sampling mercury analyser, based on CV-AAS, for which no significant \\ differences were found. Both instrumental techniques were also validated by recoveries of spikes at \\ various stages of the procedures and by the analysis of rice flour CRMs (NIST SRM 1568a and NIST SRM \\ 1568 , containing $5.8 \mu \mathrm{g} \mathrm{kg}^{-1} \mathrm{Hg}$ and $6.0 \mu \mathrm{g} \mathrm{kg}^{-1}$, respectively). Recoveries between 80 and $120 \%$ were \\ obtained and the concentrations measured in the CRM 1568a were not significantly different from the \\ certified value.
}

\section{Introduction}

Mercury and its compounds are highly toxic and are of major concern due to their widespread distribution in the environment and the ability of living systems to accumulate them within the food chain. ${ }^{1-4}$ The toxicity of mercury depends on its chemical form; the organic forms of mercury, such as methylmercury, are commonly considered more dangerous than the inorganic forms because they are fat-soluble and more easily incorporated into tissues, thus facilitating the uptake of mercury by the organism. ${ }^{5-7}$ Considering the implications of toxic mercury exposure to humans, especially methylmercury, the Joint Food and Agriculture Organization/World Health Organization (FAO/WHO) Expert Committee on Food Additives and Contaminants (JECFA) has revised its former provisional tolerable weekly intake (PTWI) of $3.3 \mu \mathrm{g} \mathrm{kg} \mathrm{kg}^{-1}$ body weight (bw) of methylmercury to $1.6 \mu \mathrm{g} \mathrm{kg}^{-1} \mathrm{bw}^{8}$ For inorganic mercury, the JECFA has set a PTWI of $4 \mu \mathrm{g} \mathrm{kg}^{-1} \mathrm{bw}$, which they consider is also applicable to total mercury exposure from other foods besides fish and shellfish. ${ }^{9}$

Environmental contamination by mercury and its compounds, arising mainly from industrial pollution, ${ }^{\mathbf{1 0} 11}$ can

${ }^{a}$ Department of Chemistry, University of Massachusetts, Amherst, MA 01003, USA. E-mail: tyson@chem.umass.edu; Fax: +1 413-545-4490; Tel: +1 413-545-0195

${ }^{b}$ Department of Chemistry, Naresuan University, Phitsanulok, Thailand cause an increase in the toxicity of rice. The determination of mercury in rice samples, however, is a topic that has not been widely researched. According to the 2011 and 2012 Atomic Spectrometry Updates on elemental speciation, ${ }^{12,13}$ the majority of mercury studies, both past and present, have focused mainly on the determination of mercury in biological samples, particularly fish and seafood, which tend to have significantly higher concentrations of mercury than rice, while recent studies performed on rice have mostly been for the determination of arsenic or selenium. ${ }^{14-17}$

Previous studies of the determination of mercury in rice include a report by Al-Saleh and Shinwari ${ }^{18}$ describing a method for the determination of cadmium, lead, and mercury in rice grain from five different countries by AAS after acid digestion, although the results are largely inconclusive regarding the mercury content in rice because of small sample size and large uncertainty; for example, of the two rice samples tested from the United States, the results ranged from 3.8 to $43.5 \mu \mathrm{g} \mathrm{kg}{ }^{-1}$, whereas the mean value \pm the standard deviation of four rice samples from Thailand was reported as $1.8 \pm 1.8 \mu \mathrm{g} \mathrm{kg}^{-1}$. Lin et al. ${ }^{19}$ successfully determined inorganic, methyl, and ethyl mercury compounds by coupling LC to vapour generation ICPMS after a rapid and simple microwave extraction procedure. Chen and Jiang $^{20}$ utilised flow injection chemical vapour generation with ICP-MS for the determination of arsenic, cadmium, and mercury in cereals and a rice flour reference 
material using a slurry sampling technique to avoid a dissolution or mineralisation step.

Plasma source-MS has become an especially attractive analytical technique for trace elemental detection because of its unique, multi-element capabilities with exceptional sensitivity, and isotope ratio measurements. ${ }^{21,22}$ While it is of particular interest to accurately analyse and monitor rice samples for their total mercury content, there are several challenges associated with the determination of mercury in solid samples by conventional ICP-MS, particularly when the mercury is present at concentrations such that the concentration in the solution after sample preparation is near that of the instrumental detection limit $\left(0.016 \mu \mathrm{g} \mathrm{L}^{-1}\right),{ }^{23}$ as it is in the case with rice grain. One challenge is the choice of sample dissolution technique in which the various mercury species are to be solubilised as the inorganic mercury ${ }^{2+}$ ion..$^{22,24}$ It is well known that mercury is easily volatilised, so samples cannot be heated to high temperatures without analyte loss and this complicates the use of microwave-assisted digestion and hotplate digestions. ${ }^{25}$ Losses equivalent to a few $\mu \mathrm{g} \mathrm{kg}{ }^{-1}$ of mercury are of little consequence when dealing with samples containing a few $\mathrm{mg}$ $\mathrm{kg}^{-1}$, but that same loss for samples containing only a few $\mu \mathrm{g} \mathrm{kg}{ }^{-1}$ mercury to begin with, is much more serious. Although acid extractions may be performed without heating, they may be less efficient and are more labour-intensive.

The other major problem encountered in the determination of total mercury by conventional ICP-MS is the severe memory effect that results in long washout times, as the mercury adheres to the walls of the sample introduction system. ${ }^{26,27}$ Several research groups have proposed procedures to alleviate this problem. Entwisle ${ }^{28}$ reported the benefits of offline addition of gold to all standards, samples, and rinse solutions. Mahar et al. have also shown that adding gold as an internal standard improves accuracy and precision. ${ }^{29}$ Woller et $a .^{30}$ added a surfactant, Triton X-100, and a complexing agent, EDTA, in the analysis of sediments by flow injection ICP-MS. Harrington et $a l^{31}$ decreased carryover by adding the sulfur-containing compound 2-mercaptoethanol (2-ME) to the carrier solution in a flow injection system. Several research groups have shown the benefits of the addition of thiols such as 2-ME, dithiothreitol (DTT) and L-cysteine, which are thought to react with mercury via the sulfur atom of the thiol group. ${ }^{27,32,33}$ Campbell et al. decreased the memory effect by the addition of salts, ${ }^{26}$ but such compounds can deposit onto and clog the cones of the plasma-source mass spectrometer, decreasing the sensitivity. ${ }^{27}$

We have developed and validated a new method for the determination of low concentrations of total mercury in rice grain by conventional ICP-MS, in which L-cysteine improved washout and stabilised the signals of both the mercury and the gold internal standard (added online). The estimated detection limit, based on three standard deviations of the blank concentration, is $0.015 \mu \mathrm{g} \mathrm{L} \mathrm{L}^{-1}$. Because this new method works with the standard sample introduction system for a plasma-source mass spectrometer, it could be of particular interest to laboratories that have a need for occasional mercury determinations, and as such, may not possess a separate instrument for mercury determinations or a high-throughput introduction system.

\section{Experimental}

\subsection{Instrumentation}

All samples were analysed with a PerkinElmer SCIEX (Shelton, CT) ELAN 6100 plasma-source mass spectrometer. Samples for the microwave-assisted digestion procedure were prepared using Teflon vessels in conjunction with a CEM Corporation (Matthews, NC) MARSXpress microwave system, Model 230/6. As part of the validation procedures, samples were also analysed by a PerkinElmer, Inc. (Shelton, CT) SMS 100 automated mercury analyser, which operates by CV-AAS and is capable of analysing solid samples. A sonicator bath from E/MC Corp., division of RAI Research Co. (Hauppauge, NY) Model 450 Ultrasonic Cleaner and a Fisher Scientific (Pittsburgh, PA) Centrific Model 225 Benchtop Centrifuge were also available. Rice samples were ground with either a Hamilton Beach Brands, Inc., Custom Grind 15 Cup Coffee Grinder (Washington, NC) or a Krups Fast Touch Coffee Grinder (Millville, NJ). Samples were filtered through Whatman Inc. Puradisc $0.20 \mu \mathrm{m}$ PES filter media (Florham Park, NJ). Internal standard solution was added online at a T-junction, located between the pump and the nebuliser. Instrumental conditions and other experimental parameters are shown in Table 1.

\subsection{Reagents and sample materials}

All solutions were prepared using $>18 \mathrm{M} \Omega \mathrm{cm}$ deionised (DI) distilled water from a Barnstead E-pure system (Bedford, MA). Certified ACS Plus nitric acid was purchased from Fisher Scientific (Fair Lawn, NJ). Mercury standards were prepared from a PerkinElmer, Inc. (Shelton, CT) $10 \mathrm{mg} \mathrm{L}^{-1}$ atomic spectroscopy standard. L-Cysteine (97\%) was purchased from Sigma-Aldrich, Inc. (St. Louis, MO). The internal standard was prepared from a gold $1000 \mathrm{mg} \mathrm{\textrm {L } ^ { - 1 }}$ Baker Instra-Analysed Reagent solution from Mallinckrodt Baker, Inc. (Phillipsburg, NJ). Rice flour CRMs NIST 1568 and NIST 1568a, as well as Trace Elements in Spinach Leaves NIST 1570a, were obtained from the National Institute of Standards and Technology (Gaithersburg, MD). Four different brands of rice (two white, two brown) were purchased at local grocery stores.

\subsection{Preparation of solutions and standards}

The rinse solution for the plasma-source mass spectrometer contained $1 \%$ L-cysteine, to limit the extent of carbon deposition on the cones, and $10 \%$ nitric acid, to match the approximate acid content of the samples after digestion.

Gold was chosen as the internal standard, as it is similar to mercury in both mass and ionisation energy; a $1 \mu \mathrm{g} \mathrm{L^{-1 }} \mathrm{Au}$ solution was prepared and added online at a T-junction. Spikes were added as 100-200 mg of a $50 \mu \mathrm{g} \mathrm{kg}^{-1}$ stock solution of the aqueous mercury standard to the samples, prior to digestion/ extraction. The amounts spiked were selected so that they produced between 2 and 5 times the observed concentrations of mercury in the sample solutions. The $50 \mu \mathrm{g} \mathrm{kg}^{-1}$ stock solution was also used to prepare all ICP-MS calibration standards (0, $0.25,0.50,1.0$, and $5.0 \mu \mathrm{g} \mathrm{kg}^{-1}$ mercury). The full range of standards (from 0 to $5.0 \mu \mathrm{g} \mathrm{kg}^{-1}$ ) was used when spiked 


\begin{tabular}{ll}
\hline Elan 6100 ICP-mass spectrometer & \\
RF power & $1500 \mathrm{~W}$ \\
Nebuliser gas flow & $1.01 \mathrm{~L} \mathrm{~min}^{-1}$ \\
Sample flow rate & $1.4 \mathrm{~mL} \mathrm{~min}{ }^{-1}$ \\
Sample pump tubing & Black/black $(0.76 \mathrm{~mm} \mathrm{id})$ \\
Internal standard flow rate & $0.4 \mathrm{~mL} \mathrm{~min}{ }^{-1}$ \\
Internal standard pump tubing & Orange/green $(0.38 \mathrm{~mm} \mathrm{id})$ \\
Nebuliser & GemTip Cross-Flow II \\
Spray chamber & Scott \\
Detector mode & Dual mode \\
Sampler/skimmer cones & Nickel \\
Scanning mode & Peak hopping \\
Dwell time & $100 \mathrm{~ms}$ per point \\
Number of sweeps per reading & 10 \\
Number of reads per replicate & 5 \\
Number of replicates & 5 \\
Isotopes monitored & $202 \mathrm{Hg},{ }^{197} \mathrm{Au}$
\end{tabular}

$\begin{array}{ll}\text { MARSXpress microwave system } & \\ \text { Vessels } & \text { XPress vessels, } 75 \mathrm{~mL} \text { Teflon } \\ \text { Power } & 400 \mathrm{~W} \\ \text { Percent power operation } & 100 \% \\ \text { Ramp time } & 20 \mathrm{~min} \\ \text { Maximum temperature } & 100{ }^{\circ} \mathrm{C} \\ \text { Hold time } & 20 \mathrm{~min} \\ \text { Cool down time } & 60 \mathrm{~min}\end{array}$

SMS 100 mercury analyser

Sample boats

Drying temperature $\quad 400{ }^{\circ} \mathrm{C}$

Drying time $200 \mathrm{~s}$

Decomposition temperature $\quad 800{ }^{\circ} \mathrm{C}$

Decomposition time $200 \mathrm{~s}$

Catalyst temperature $\quad 600{ }^{\circ} \mathrm{C}$

Catalyst wait period $\quad 60 \mathrm{~s}$

Gold trap temperature $\quad 600{ }^{\circ} \mathrm{C}$

Gold trap time $\quad 30 \mathrm{~s}$

Measurement time $100 \mathrm{~s}$

Oxidant gas $\quad \mathrm{O}_{2}$

Oxidant gas flow $\quad 350 \mathrm{~mL} \mathrm{~min}^{-1}$

solutions were included in the experiment; otherwise, in the absence of spikes, only standards covering the range 0 to 1.0 $\mu \mathrm{g} \mathrm{kg}{ }^{-1}$ were used. All solutions were made fresh daily.

\subsection{Rice sample preparation}

Approximately $10 \mathrm{~g}$ of the rice samples were ground for 30-60 s into a relatively uniform powder $(<500 \mu \mathrm{m}$ diameter $)$ to ensure representative sampling. Samples were stored in the refrigerator in polypropylene centrifuge tubes until needed. Samples were equilibrated with the laboratory humidity and analysed "as received."

\subsection{Data analysis}

All calculations were performed with Microsoft Excel. Calibration functions were fitted by unweighted linear least squares regression. In the case of the microwave-assisted digestion experiments, the average mercury contribution from the reagent blanks was subtracted. This was not necessary for the acid extraction procedure, as the reagent blanks were very close to the calibration blank.

\subsection{Microwave-assisted digestion procedure}

Rice samples $(500 \mathrm{mg}$ ) were accurately weighed directly into the microwave vessels, followed by approximately $200 \mathrm{mg}$ of L-cysteine. Any spikes, prepared from the stock mercury solution were added at this stage. L-Cysteine reacts violently with nitric acid, and even more quickly in the presence of organic rice matter, and so this addition should be performed in a hood; deionised water $(3 \mathrm{~mL})$ and concentrated nitric acid $(3 \mathrm{~mL})$ were carefully added, in that order, to lessen the severity of the reaction. After the reaction, the vessels were shaken vigorously and left in the hood overnight, uncapped, to allow for predigestion. Reagent blanks were prepared in the same way. Calibration standards were prepared on the same day as the samples, with approximately $10 \%$ nitric acid and 1\% L-cysteine; although the standards were not to be digested in the microwave the next day and were instead prepared in polystyrene centrifuge tubes, they, too, were left uncapped in the hood overnight. Samples and calibration standards without L-cysteine were also prepared according to the same procedure.

The following day, the vessels were capped and heated by microwave radiation according to the parameters shown in Table 1. This procedure is gentler than most microwave programs to prevent mercury loss through venting of the vessels, although the samples are not completely dissolved. The samples were diluted to a total mass of 14-15 g with DI water and filtered twice to remove any remaining particles; standards were filtered only once. All samples and standards were analysed by ICP-MS on the same day. This procedure was validated by the analysis of spikes and rice flour SRM 1568a.

\subsection{Acid extraction procedure}

The acid extraction procedure was modified from that of Shao et $a l .{ }^{34}$ Ground rice samples (500 $\mathrm{mg}$, accurately weighed) were placed into $15 \mathrm{~mL}$ polystyrene centrifuge tubes along with approximately $250 \mathrm{mg}$ L-cysteine. Any spikes were added at this stage, and then the extractant mixture was added $(5 \mathrm{~mL}$ of DI water and $2 \mathrm{~mL}$ of concentrated $\mathrm{HNO}_{3}$ ). The centrifuge tubes were sonicated in a water bath for $60 \mathrm{~min}$ and then centrifuged at $3500 \mathrm{rpm}$ for $5 \mathrm{~min}$. The supernatant was decanted into a 50 $\mathrm{mL}$ polypropylene centrifuge tube, and the residue was extracted once more as described above. Afterwards, the two supernatant portions were added together and diluted to $25 \mathrm{~mL}$ with DI water to make the final concentration of $\mathrm{L}_{\text {-cysteine approxi- }}$ mately $1 \%$. Calibration standards were prepared in the same manner. In a comparative study, samples and calibration standards without L-cysteine were prepared according to the same procedure. All samples and standards were filtered, then analysed by ICP-MS on the same day. This procedure was also validated by the analysis of spikes and rice flour SRM 1568a.

\subsection{Procedure for the mercury analyser}

The mercury analyser was calibrated using varied masses of NIST rice flour SRM 1568a. Ground rice samples (approximately 
$150 \mathrm{mg}$ ) were accurately weighed into the sample boats and analysed with the program given in Table 1 . Results were validated with the NIST rice flour SRM 1568, which is a different lot of the same rice flour SRM, as well as by spiking with the rice flour SRM 1568a. The instrument was also calibrated with NIST CRM 1570a, trace elements in spinach leaves.

\section{Method development}

\subsection{Preliminary experiments}

Preliminary experiments were performed to evaluate and optimise the different potential methods and techniques. The figure of merit was primarily accuracy of the analysis of the rice RMs, but results with precisions greater than 10\% RSD were considered suboptimal. Experimental factors were considered to be independent, and so the single-cycle alternating variable search was adopted. For the minimisation of memory effect, several additives to both rinse solutions and samples were evaluated including 1-2\% NaCl, gold, and L-cysteine. The washin and wash-out profiles of a $10 \mu \mathrm{g} \mathrm{kg}{ }^{-1}$ mercury standard were compared for two different compositions of the sample and rinse solutions normalised to the maximum signal intensity. The $\mathrm{Au}$ internal standard was not used in this particular experiment. The blank solution in each experiment was run prior to the mercury standard solution, in order to obtain information on the background mercury signal at baseline. For the microwave-assisted digestion the effects of final dilution volumes, of various microwave temperatures, and of different reagents and reagent concentrations were evaluated. Sample mass was fixed at $500 \mathrm{mg}$ as recommended by PerkinElmer, Inc. and the CEM Corporation; larger quantities were originally tried, but greater difficulty in filtering the samples and mercury loss due to venting during microwave digestion as a result of a higher internal pressure was encountered. For the acid extraction, the effects of varying sonication and centrifugation times on $500 \mathrm{mg}$ of sample were studied. The effect of sonication time on (a) the value obtained for the concentration of the certified value of the rice flour SRM 1568a (expressed as a percentage of the certificate value) and (b) recoveries of aqueous spikes (both SRM samples that had been spiked prior to the extraction procedure and those that had been spiked after the extraction) was evaluated. The differences in the results between samples prepared both with and without the addition of 1\% L-cysteine were examined for both the microwave-assisted digestion and acid extraction methods. For the mercury analyser, calibration with aqueous standards was compared with that with solid standards.

\subsection{Method validation}

The methods involving microwave-assisted digestion and acid extraction were validated by the analysis of spiked samples, as well as a CRM, rice flour SRM 1568a. The spikes were added as aqueous standards to the samples, prior to digestion/extraction, whereas the RMs were analysed as if they were rice samples. The spikes were delivered by adding approximately 100-200 $\mathrm{mg}$ of a $50 \mu \mathrm{g} \mathrm{kg}{ }^{-1}$ stock solution of the aqueous mercury standard.
Additionally, samples were analysed by a method involving a different instrumental technique, CV-AAS. The performance of this method was also evaluated by the analysis of spiked samples and a CRM, rice flour SRM 1568.

\section{Results and discussion}

\subsection{Minimisation of memory effect}

Of the three additives examined for their potential to improve the washout efficiency, only L-cysteine showed noticeable improvements and was, therefore, chosen for the remainder of the experiments. For solutions containing $2 \%$ nitric acid, approximately $3.4 \mathrm{~min}$ elapsed before steady state was reached (i.e. the signal fluctuated only $\pm 5 \%$ ), and 113 min were needed for the signal to return to the original baseline value (i.e. the value obtained when a blank solution was continuously nebulised prior to switching to a standard). For solutions containing $10 \%$ nitric acid and 1\% L-cysteine, both the wash-in and wash-out times were significantly decreased to $1.3 \mathrm{~min}$ and 13.2 min, respectively. A signal "spike" was observed around $\mathbf{1 7 . 5}$ min for the solution containing $2 \%$ nitric acid; this effect was also observed by Li et al. ${ }^{27}$ and was explained by the wash-out of mercury that had been accumulating on the walls of the spray chamber.

\subsection{Microwave-assisted digestion procedure}

Samples were diluted only to 14-15 $\mathrm{g}$ because it was found that greater dilutions produced mercury concentrations below the instrumental detection limit. The optimal temperature for the digestion was set to not exceed $100{ }^{\circ} \mathrm{C}$ because anything higher resulted in analyte loss. Digestion with hydrogen peroxide and nitric acid gave low recoveries due to the venting of the vessels, whereas digestion with 5-6 mL of concentrated nitric acid gave unacceptable accuracy and precision. However, it was found that digestion with $3 \mathrm{~mL}$ nitric acid and $3 \mathrm{~mL}$ DI water solubilised the mercury (although filtration was required to remove the residual matrix), while producing an acid concentration that did not adversely affect the precision of the measurements. Table 2 shows the results for the four rice samples, with L-cysteine, prepared by microwave-assisted digestion. A comparison (not shown) was made between samples that contained approximately 1\% L-cysteine added prior to digestion, versus samples that did not contain L-cysteine. Statistical analysis, using Student's $t$-test, indicates that there is no significant difference between the two sample means at the $95 \%$ confidence level.

\subsection{Acid extraction procedure}

The optimum sonication time was $60 \mathrm{~min}$ and the optimum centrifugation time was approximately $5 \mathrm{~min}$. The results for the samples prepared by the acid extraction procedure are also given in Table 2. The differences between samples to which L-cysteine had been added and those without L-cysteine (results not shown) were examined. With the exception of white rice 1 , all of the results were significantly different on the basis of a $t$-test at the $95 \%$ confidence level indicating that the addition of 


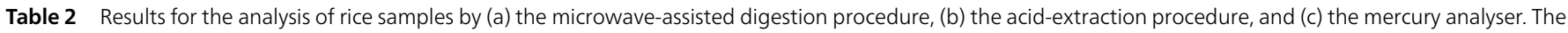

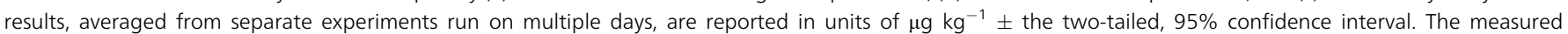

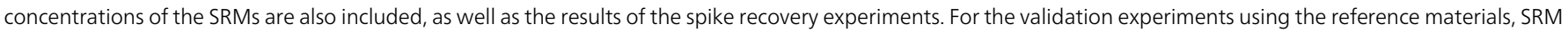

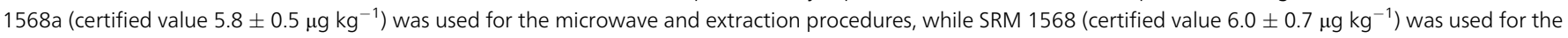
mercury analyser. The \pm term for the reference materials is the $95 \%$ confidence interval plus additional allowance for systematic error among the methods used

\begin{tabular}{lccc}
\hline Sample ID & Microwave & Extraction & Mercury analyser \\
\hline SRM & $5.8 \pm 0.7(N=4)$ & $5.1 \pm 0.4(N=7)$ & $6.7 \pm 0.1(N=5)$ \\
Spike recovery experiments, \% & $94.5 \pm 16(N=4)$ & $99.3 \pm 0.71(N=3)$ & $103 \pm 8.8(N=6)$ \\
White rice 1 & $6.31 \pm 1.4(N=3)$ & $3.61 \pm 1.3(N=3)$ & $6.29 \pm 0.32(N=10)$ \\
White rice 2 & $4.39 \pm 0.57(N=5)$ & $5.77 \pm 0.32(N=4)$ & $3.25 \pm 0.11(N=7)$ \\
Brown rice 1 & $4.06 \pm 1.4(N=6)$ & $2.86 \pm 0.29(N=7)$ & $5.95 \pm 0.13(N=8)$ \\
Brown rice 2 & $4.15 \pm 0.25(N=5)$ & $5.49 \pm 0.42(N=5)$ & $3.59 \pm 0.11(N=7)$ \\
& & &
\end{tabular}

L-cysteine makes more of a difference to the acid extraction method than it does to the microwave digestion method. A comparison of the results for the microwave plus L-cysteine procedure with those of the extraction plus L-cysteine procedure by a paired $t$-test shows that these two methods do not yield significantly different results. However, a comparison of the results for individual samples shows significant differences at the $95 \%$ confidence level (though not at the 99.9\% level), with the exception of brown rice 1 and SRM 1568a. The extraction procedure was far more labour-intensive and time-consuming than the microwave procedure; however, despite its drawbacks, the acid extraction method yielded acceptable results for the concentrations of mercury in rice at single-digit, $\mu \mathrm{g} \mathrm{kg}^{-1}$ values, and could be considered to be a viable alternative if a microwave system is not available.

\subsection{Addition of L-cysteine to samples}

The addition of L-cysteine to the samples improved washout times and may have prevented the loss of analyte during the microwave digestion method. The addition of nitric acid to L-cysteine in the presence of organic rice matrix causes (a) the formation of what appears to be colloidal sulfur particles, and (b) gas evolution. Statistical analysis showed that there was no significant difference (at 95\% confidence) between the results obtained with the addition of L-cysteine and those obtained without L-cysteine. The addition of L-cysteine played a greater role in the acid extraction method, as statistical analysis (95\% confidence) showed that the results for samples prepared with L-cysteine were significantly higher than the results for those prepared without, with the exception of white rice 1 and SRM 1568a. Furthermore, the L-cysteine may also contribute to the stabilisation of the Au signal, as it was often observed that poor accuracy and precision would arise from fluctuations in the internal standard signal rather than in the mercury signal, particularly when L-cysteine was not present.

\subsection{Mercury analyser}

Preliminary experiments involving analysis of rice flour SRM 1568 (certified value $6.0 \pm 0.7 \mu \mathrm{g} \mathrm{kg}^{-1}$ ) against a calibration with aqueous standards yielded results that were consistently about $50 \%$ too high. For instance, the measured value for SRM 1568 was $9.3 \pm 0.1 \mu \mathrm{g} \mathrm{kg}{ }^{-1}$ (95\% confidence interval). When the mercury analyser was calibrated with a matrix-matched calibration standard, namely rice flour SRM 1568a (certified value $5.8 \pm 0.5 \mu \mathrm{g} \mathrm{kg}^{-1}$ ), the results, $6.7 \pm 0.1 \mu \mathrm{g} \mathrm{kg}{ }^{-1}$, were in agreement with the certified value. Calibrating by this method also yielded spike recoveries (spiked with SRM 1568a rice flour) that were not significantly different from $100 \%$.

These preliminary experiments prompted a comparison study between the slopes of three calibrations materials: (a) an aqueous curve, created by using various masses of a 12 and 100 $\mu \mathrm{g} \mathrm{kg}^{-1}$ mercury solution and yielding an unweighted linear least squares regression equation of $y=8.74 \times 10^{-3} \mathrm{~m}+2.5 \times$ $10^{-4}$ (where $y$ is the instrument response in absorbance and $m$ is the mercury mass in ng), (b) a curve prepared from the responses to known masses of rice flour SRM 1568a and yielding an equation of $y=1.22 \times 10^{-2} \mathrm{~m}+1.6 \times 10^{-4}$, and (c) a curve created from SRM 1570a trace elements in spinach leaves (certified value of $30 \mu \mathrm{g} \mathrm{kg}{ }^{-1}$ ), which produced an equation for the line of $y=1.08 \times 10^{-2} m+1.8 \times 10^{-4}$. The significant difference between instrument response factors for these three calibration materials clearly demonstrates the need for matrix matching the standards to the samples being analysed. The rice flour SRM 1568a was used to calibrate the mercury analyser, and the results for the rice sample analyses are shown in Table 2.

\subsection{Method validation}

The results of the method validation experiments are shown in Table 2. For the results of each method, the 95\% confidence interval is given for both the values obtained for the concentrations of the rice flour SRMs and the percent recovery of the spikes. Validation studies for the microwave method were performed using the rice flour SRM 1568a, as well as with aqueous mercury spikes that were added prior to digestion. With the exception of the recoveries of spikes into the samples prepared without the addition of L-cysteine (results not shown), the recoveries were not significantly different from $100 \%$. The values obtained for the concentration in the SRM were not significantly different from the certificate value.

For the acid extraction procedure without the addition of L-cysteine (results not shown), the spike recoveries were high, but the value for the concentration in the SRM was not significantly different from the certificate value (but only because the 
uncertainly was relatively high). For the procedure with the addition of L-cysteine, the spike recoveries were not significantly different from $100 \%$, but the value obtained for the concentration in the SRM was low. A paired $t$-test shows that there is no significant difference between the set of results obtained by the acid extraction method and the corresponding set obtained by the mercury analyser.

For the mercury analyser method, spike recoveries were not significantly different from $100 \%$, and the concentration measured in the SRM was in agreement with the certified value. The results obtained by the methods involving plasma-source mass spectrometry were further validated by comparison with the results obtained by the mercury analyser, as summarised in Table 2. A comparison of the results obtained by the microwave plus L-cysteine method with the results for the mercury analyser for the four samples, by a paired $t$-test, shows no significant difference at the $95 \%$ confidence level.

\section{Conclusions}

A new method of microwave-assisted digestion has been developed for the determination of low concentrations of total mercury in rice grain by conventional ICP-MS. The analysis was made possible by the addition of $1 \%$ L-cysteine to all standards, samples, and rinse solutions, as well as the online addition of a gold internal standard. The addition of L-cysteine and gold improved accuracy and precision while decreasing the memory effect. The L-cysteine also contributed to the stabilisation of the gold signal, as well as helping to prevent the mercury from adhering to the sample introduction system. A comparison between microwave digestion and acid extraction showed that the analysis could be performed without a microwave digestion system, though an ultrasonic bath is needed.

It is unclear if the amount of mercury reported in the rice samples under study should be cause for concern; while these concentrations fall below the specified PTWI, more studies on the long-term exposure to small amounts of mercury are perhaps needed, and provisional weekly or daily intake limits need to reflect multiple exposure pathways and different chemical forms. ${ }^{35,36}$ Speciation studies should also be performed in the future on some of the more common rice brands sold in the U.S., in order to identify the predominant species present and thereby have more data available with which to assess risk. Furthermore, there appears to be no information available on the homogeneity of the rice grains in a bag with respect to trace element content. This information is needed, not just for the study of mercury in rice but also for studies of elements of potentially much greater concern, such as arsenic and selenium.

This new method, which only requires a plasma-source mass spectrometer and standard sample introduction system, may be of particular interest to labs that have a need for occasional mercury determinations, and as such, may not own a separate instrument for mercury determinations or a high-throughput sample introduction system. Furthermore, the method is capable of determining low concentrations that are relevant to the maximum allowable daily or weekly intake levels, and could possibly be applied to the routine monitoring of a variety of foodstuffs (especially other grains and cereals) for the mercury content as well as for a variety of other trace elements of interest, such as arsenic and selenium. Studies relating to the determination of arsenic in rice and to selenium in dietary supplements are in progress and will be reported shortly.

\section{Acknowledgements}

We would like to express our deepest gratitude to PerkinElmer, Inc., for their financial support for L. D-H, and particularly to Laura Thompson for her support and guidance. We would also like to thank PerkinElmer, Inc. for access to the SMS 100 Automated mercury Analyzer. Financial support for S. W. from Naresuan University is gratefully acknowledged.

\section{References}

1 E. M. Sunderland and G. L. Chmura, Environ. Pollut., 2000, 110, 297-306.

2 Q. Wang, W. G. Shen and Z. W. Ma, Environ. Sci. Technol., 2000, 34, 2711-2713.

3 É. M. M. Flores, B. Welz and A. Curtius, Spectrochim. Acta, Part B, 2001, 56, 1605-1614.

4 H. Y. Zhou and M. H. Wong, Water Res., 2000, 34, 4234-4242.

5 I. Serafimovski, I. Karadjova, T. Stafilov and J. Cvetković, Microchem. J., 2008, 89, 42-47.

6 U. Förstner and G. T. W. Wittmann, in Metal Pollution in the Aquatic Environment, Springer-Verlag, Berlin, 2nd edn, 1981.

7 O. V. Shuvaeva, M. A. Gustaytis and G. N. Anoshin, Anal. Chim. Acta, 2008, 621, 148-154.

8 Summary and conclusions of the sixty-seventh meeting of the Joint FAO/WHO Expert Committee on Food Additives (JECFA), July 2006.

9 Safety evaluation of certain contaminants in food, seventyseventh meeting of the Joint FAO/WHO Expert Committee on Food Additives (JECFA), WHO Food Additive Series 63, 2011.

10 E. G. Pacyna, J. M. Pacyna, F. Steenhuisen and S. Wilson, Atmos. Environ., 2006, 40, 4048-4063.

11 A. T. Reis, S. M. Rodrigues, C. Araújo, J. P. Coelho, E. Pereira and A. C. Duarte, Sci. Total Environ., 2009, 407, 2689-2700.

12 C. F. Harrington, R. Clough, L. R. Drennan-Harris, S. J. Hill and J. F. Tyson, J. Anal. At. Spectrom., 2011, 26, 1561-1595.

13 C. F. Harrington, R. Clough, L. R. Drennan-Harris, S. J. Hill and J. F. Tyson, J. Anal. At. Spectrom., 2012, 27, 1185-1224.

14 J. L. Guzmán Mar, L. Hinojosa Reyes, G. M. Mizanur Rahman and H. M. Skip Kingston, J. Agric. Food Chem., 2009, 57, 3005-3013.

15 C.-Y. Tsai and S.-J. Jiang, Anal. Sci., 2011, 27, 271-276.

16 D. Gilbert-Diamond, K. L. Cottingham, J. F. Gruber, T. Punshon, V. Sayarath, A. J. Gandolfi, E. R. Baker, B. P. Jackson, C. L. Folt and M. R. Karagas, Proc. Natl. Acad. Sci. U. S. A., 2011, 108, 20656-20660.

17 A. Taylor, S. Branch, M. P. Day, M. Patriarca and M. White, J. Anal. At. Spectrom., 2010, 25, 453-492. 
18 I. Al-Saleh and N. Shinwari, Biol. Trace Elem. Res., 2001, 83, 91-96.

19 L. Y. Lin, L. F. Chang and S. J. Jiang, J. Agric. Food Chem., 2008, 56, 6868-6872.

20 F. Y. Chen and S. J. Jiang, J. Agric. Food Chem., 2009, 57, 6564-6569.

21 R. G. Smith, Anal. Chem., 1993, 65, 2485-2488.

22 Z. Arslan, N. Ertas, J. F. Tyson, P. C. Uden and E. R. Denoyer, Fresenius' J. Anal. Chem., 2000, 366, 273-282.

23 Detection limits, Atomic Spectroscopy, A Guide to Selecting the Appropriate Technique and System, Perkin Elmer, p. 12, http:// www.perkinelmer.com/Technologies/Atomic-Spectroscopy/ cat1/NAV_04_TCH_Technologies_001/cat2/NAV_TCH_Atomic \%20Spectroscopy_001, accessed November $8^{\text {th }} 2012$.

24 S. B. Adeloju, H. S. Dhindsa and R. T. Tandon, Anal. Chim. Acta, 1994, 285, 359-364.

25 C. Lomonte, D. Gregory, A. J. M. Baker and S. D. Kolev, Chemosphere, 2008, 72, 1420-1424.

26 M. J. Campbell, G. Vermeir, R. Dams and P. Quevauviller, J. Anal. At. Spectrom., 1992, 7, 617-621.
27 Y. Li, C. Chen, B. Li, J. Sun, J. Wang, Y. Gao, Y. Zhao and Z. Chai, J. Anal. At. Spectrom., 2006, 21, 9496.

28 J. Entwisle, Am. Lab., 2004, 36, 11-14.

29 M. Mahar, J. F. Tyson, K. Neubauer and Z. Grosser, J. Anal. At. Spectrom., 2008, 23, 1204-1213.

30 A. Woller, H. Garraud, F. Martin, O. F. X. Donard and P. Fodor, J. Anal. At. Spectrom., 1997, 12, 53-56.

31 C. F. Harrington, S. A. Merson and T. M. D'Silva, Anal. Chim. Acta, 2004, 505, 247-254.

32 H. Zhong and W. X. Wang, Environ. Sci. Technol., 2006, 40, 6181-6186.

33 R. T. Drexel, M. Haitzer, J. N. Ryan, G. R. Aiken and K. L. Nagy, Environ. Sci. Technol., 2002, 36, 40584064.

34 L. J. Shao, W. E. Gan and Q. D. Su, Anal. Chim. Acta, 2006, 562, 128-133.

35 K. Francesconi, Analyst, 2007, 132, 17-20.

36 A. A. Meharg and A. Raab, Environ. Sci. Technol., 2010, 44, 4395-4399. 